\title{
Outcome of staged reconstructive surgery for hypoplastic left heart syndrome following antenatal diagnosis
}

\author{
R Andrews, R Tulloh, G Sharland, J Simpson, S Rollings, E Baker, S Qureshi, \\ E Rosenthal, C Austin, D Anderson
}

\begin{abstract}
Background and Aims-Staged reconstructive surgery has radically altered the prognosis of hypoplastic left heart syndrome (HLHS). Antenatal diagnosis allows for appropriate counselling, and time to consider treatment options. We report outcome from a centre where most cases are antenatally diagnosed and delivered on site.

Methods-Information was collated on 188 consecutive cases of HLHS between 1995 and 2000, including timing of diagnosis, outcome of pregnancy, and age and outcome at each stage of surgery. At Guy's Hospital, 174 cases were diagnosed antenatally, of whom 50 underwent surgery. Fourteen others (five diagnosed antenatally at other centres, and nine diagnosed postnatally) also underwent surgery.

Results-Survival after stage I (the Norwood operation) was $52 \%$ (33/64). Postoperative survival after stage II (the hemiFontan operation, performed in 29), and stage III (the Fontan operation, performed in 10 ), was $100 \%$. Two late deaths occurred 3 and 10 months after stage II, giving overall survival of $48 \%$ (31/64). At follow up, three children have neurological impairment, and one had poor right ventricular function necessitating cardiac transplantation.

Conclusions-Antenatal diagnosis allows informed decisions about treatment options, and facilitates preoperative care. Mortality following stage I is high, irrespective of timing of diagnosis, but medium term outcome for survivors is good. (Arch Dis Child 2001;85:474-477)
\end{abstract}

Congenital Heart

Disease, Guy's

Hospital, Guy's and St

Thomas' NHS Trust,

London, UK

$\mathrm{R}$ Andrews

R Tulloh

G Sharland

J Simpson

S Rollings

E Baker

S Qureshi

E Rosenthal

C Austin

D Anderson

Correspondence to: Dr Tulloh

Robert.Tulloh@

gstt.sthames.nhs.uk

Accepted 21 February 2001 atresia, with underdevelopment of the left ventricle, and varying degrees of hypoplasia of the aortic arch. Both pulmonary and systemic circulations are, therefore, dependent on the right ventricle, with flow to the descending aorta being provided through a patent arterial duct. Patency of the duct can be maintained with an infusion of prostaglandin $\mathrm{E}_{1}$ or $\mathrm{E}_{2}$ while the infant is stabilised prior to surgery, but if the duct is allowed to close the systemic circulation cannot be supported and the infant will die if untreated. $^{5}$

Reconstructive surgery for this condition is performed in three stages. The first stage, which has become known as the Norwood operation, is performed within the first few days of life. This consists of reconstruction of the hypoplastic aorta using the proximal main pulmonary artery, removal of the atrial septum, and insertion of a systemic to pulmonary artery shunt.

The second stage, the hemi-Fontan operation, is carried out between 4 and 12 months, and consists of an anastomosis between the superior vena cava (SVC) and the right pulmonary artery. The third stage, the Fontan operation, is performed at 3-4 years of age, and consists of an anastomosis between the inferior vena cava (IVC) and the right pulmonary artery. This series of operations allows for complete separation of the pulmonary and systemic circulations, with systemic venous drainage passing directly to the lungs, and the right ventricle acting solely as the systemic pump.

The improved prognosis of HLHS makes antenatal diagnosis all the more important, to allow the parents time to consider the treatment options, and to arrange for elective delivery in a specialist centre, if desired. It also allows for a coordinated service to be provided between fetal cardiology, neonatology, paediatric cardiology, and cardiac surgery, such that the parents can meet with the relevant specialists prior to delivery of their baby, and be shown around the unit. In order to counsel parents accurately antenatally, it is essential to understand the natural history of this condition, ${ }^{6}$ and to be able to describe survival after surgery. ${ }^{7-11}$ We report the outcome of staged reconstructive surgery for HLHS in a centre where most of the cases were antenatally diagnosed and delivered on site.

\section{Methods}

PATIENTS

Information from the fetal and surgical databases was collated on 188 consecutive patients with HLHS from the start of the Norwood 
programme at Guy's Hospital in July 1995 to July 2000. All patients included in the study had classical HLHS, defined as a right ventricular dependent circulation in association with atresia or severe hypoplasia of the aortic and mitral valves and the aortic arch, with consequent underdevelopment of the left ventricle, and were diagnosed by two dimensional echocardiography. Patients requiring a Norwood type reconstruction for variants of HLHS involving other anatomical defects such as severely unbalanced atrioventricular septal defects, or double inlet or outlet ventricles, were excluded.

Measures assessed included timing of diagnosis (ante- or postnatal), outcome of pregnancy, age at each stage of surgery, and outcome from each stage in terms of morbidity and mortality. Student's $t$ test and $\chi^{2}$ tests were used for statistical analysis.

A total of 174 cases of HLHS were diagnosed antenatally at Guy's Hospital (fig 1). Of these, $105(60 \%)$ sets of parents elected to terminate the pregnancy, five babies died in utero, two pregnancies were continuing, and there were 62 live births. Of these 62 , six families declined treatment, and the babies died at a mean age of 4.0 (SE 1.8) days.

All parents who opted for active management chose to be delivered at Guy's Hospital, although one mother subsequently went into premature labour and delivered at her local hospital, necessitating an ex utero transfer.

Six babies, whose parents opted for active management, died before surgery: two from extreme prematurity (at 23 and 25 weeks gestation respectively); one following in utero dilation of the aortic valve at 32 weeks gestation; one from severe right ventricular failure with hydrops at 34 weeks gestation; one from tamponade following balloon atrial septostomy; and one term baby who was persistently acidotic and hypotensive from birth despite full supportive measures.

Thus there were 50 babies diagnosed antenatally who underwent stage I (29\%). Fourteen other babies also underwent stage I surgery at Guy's Hospital: five diagnosed antenatally at other centres (of which three were delivered at Guy's), and nine diagnosed postnatally and transferred ex utero.

Stage I was performed on 64 babies at a mean age of 3.5 (SE 0.5) days (antenatal diagnoses 3.0 days compared with postnatal

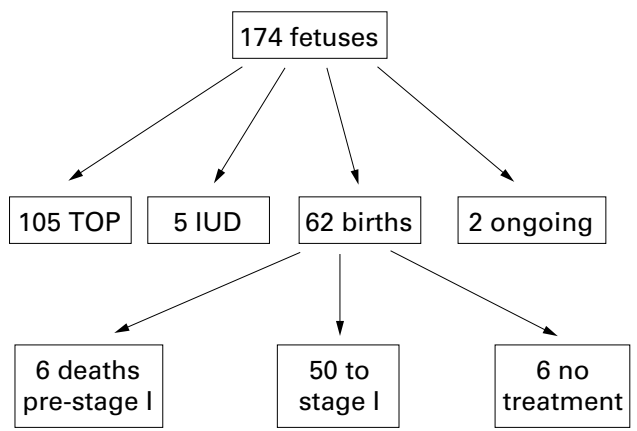

Figure 1 Outcome of 174 fetuses diagnosed antenatally at Guy's Hospital with HLHS. TOP, termination of pregnancy; IUD, intrauterine death. diagnoses 5.8 days; $\mathrm{p}<0.05)$. Stage II was performed on 29 infants at a mean age of 8.3 (0.6) months. Stage III was performed on 10 children at a mean age of 40.7 (0.8) months.

\section{OPERATIVE TECHNIQUES}

Stage I surgery consisted of three parts: construction of a neoaorta by incorporating the undersized aorta into the pulmonary trunk, using a homograft patch to enlarge it; removal of the atrial septum to provide unobstructed pulmonary venous return to the right atrium; and the creation of a systemic to pulmonary shunt between the innominate artery and the right pulmonary artery, the distal main pulmonary artery having been disconnected from the pulmonary trunk. The duct was also ligated and divided.

At stage II, a partial separation of the pulmonary and systemic circulations was created by the anastomosis of the SVC to the right pulmonary artery, with a patch sewn across the entrance of the SVC to the right atrium. The systemic to pulmonary artery shunt was also disconnected.

At stage III, complete separation of the pulmonary and systemic circulations was achieved by suturing a baffle along the lateral wall of the right atrium to create a tunnel, and by removing the patch at the lower end of the SVC, so that blood from the IVC could flow through the lateral tunnel via the distal SVC into the right pulmonary artery without mixing with blood in the body of the right atrium. A $4 \mathrm{~mm}$ fenestration was left in the baffle to allow any rise in the right sided pressures to decompress to the systemic circuit.

\section{Results}

Survival after stage I was $52 \%$ (33/64). Of the 31 deaths, 30 occurred within the first month. Three deaths occurred intraoperatively, as the babies could not be weaned off bypass, and a further 18 babies died within the first 24 hours postoperatively. Seven further deaths occurred within 1 week of surgery, and two occurred at 3 weeks. There was one death at 6 weeks.

Of the 25 deaths which occurred on the intensive care unit within a week of surgery, four were caused by intracranial bleeds, five by necrotising enterocolitis, and two shunt blockages. However the majority $(n=14)$ of the deaths occurred secondary to poor cardiac output despite maximal inotropic support. Two deaths occurred 3 weeks postoperatively following a collapse on the ward; both were caused by neoaortic arch obstruction. One death occurred at home 6 weeks postoperatively, of an unknown cause. A limited autopsy revealed no structural problems with the heart, great vessels, or shunt.

The early survival after stage II (29 cases) was $100 \%$. However there were two late deaths, at 3 and 10 months respectively. The first case presented moribund to the local casualty department following a short history of an upper respiratory tract infection, and died before he could be transferred to a paediatric intensive care unit (PICU). An autopsy was not 


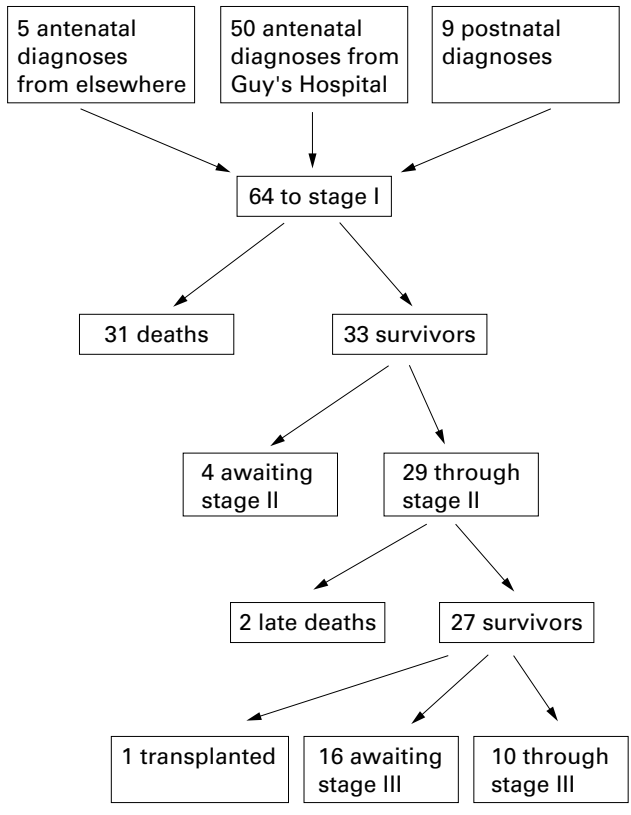

Figure 2 Outcome of 64 children with HLHS entered into the Norwood programme.

performed, and the exact cause of death was unclear.

The second child collapsed at home and was transferred via his local hospital to our PICU. He was subsequently found to have had a cerebrovascular accident, and to have poor right ventricular function. He died within hours of presentation despite full intensive care. At autopsy cerebral infarction was confirmed, although no source of embolus was seen in the heart or great vessels.

Survival following stage III (10 cases) was $100 \%$

The overall survival for the series is $48 \%$ (31/64). For babies who were diagnosed antenatally, the overall survival rate was $49 \%$ (27/55), or $44 \%$ taken from an intention to treat basis (27/61 live births); for those who were postnatally diagnosed, it was $44 \%$ (4/9). Figure 2 shows the outcome for all babies who underwent surgery.

Of the 64 babies in this series who underwent surgery, six had extracardiac or karyotypic anomalies, all of which were detected antenatally. Table 1 shows their diagnoses and outcome.

At follow up (median 35 months, range 3-63), three children have a degree of neurological impairment. The first was born with a left congenital diaphragmatic hernia, which was repaired at the same time as his stage I operation. He made a good recovery postoperatively, but was subsequently found to have congenital aqueduct stenosis causing hydrocephalus, which required a ventriculoperitoneal shunt. $\mathrm{He}$ is now systemically well although mildly developmentally delayed, and has successfully undergone stage III.

The second child developed features of an autistic spectrum disorder at 2 years of age. $\mathrm{He}$ has successfully undergone stage III, and at 4 years has good comprehension, although his speech is delayed. His younger brother, who has a structurally normal heart, has since been diagnosed with the same condition.

The third child was born with Pierre Robin sequence, a cleft palate, and severe left talipes equinovarus, which have required surgery. Initially he underwent balloon dilatation of his aortic valve for critical aortic stenosis, progressing to a Norwood operation at 9 days of age, when it had become evident that he was unsuitable for biventricular repair. His postoperative course was complicated by frequent seizures, although these have subsequently resolved. He is now awaiting stage III, but has moderate to severe developmental delay, with impaired hearing, a divergent squint, and a degree of cortical visual loss.

In addition, one child had poor right ventricular function with severe tricuspid regurgitation following stage II, requiring an elective tricuspid valve repair. The tricuspid regurgitation was less severe following this procedure, but the right ventricular function remained poor. This patient has recently undergone successful cardiac transplantation.

\section{Discussion}

Staged reconstructive surgery provides effective palliation for children with HLHS, and provides an alternative to heart transplantation in the neonatal period, the use of which is limited by shortage of donors. The availability of antenatal diagnosis and active postnatal treatment of HLHS has radically altered the outlook for affected babies in our region. The Norwood programme at Guy's Hospital was initiated, at least in part, by increasing parental demand for active management of HLHS, in response to improving outcomes around the world. Previously a uniformly fatal condition, our data show overall survival of over $50 \%$ for those entered into the programme.

Antenatal diagnosis of HLHS has obvious benefits for parents, not least in giving them time in which to come to terms with the diagnosis, and make informed decisions about the

Table 1 Outcome for babies with extracardiac and karyotypic anomalies

\begin{tabular}{lllll}
\hline Case no. & Diagnosis & Karyotype & Surgical stage & Outcome \\
\hline 1 & CDH, aqueduct stenosis & $46 \mathrm{XY}$ & Post stage III & Well, mild developmental delay \\
2 & Pierre Robin sequence, talipes & $46 \mathrm{XY}$ & $\begin{array}{l}\text { Post stage II } \\
\text { Well, global developmental delay }\end{array}$ \\
3 & CDH & $46 \mathrm{XX}$ & $\begin{array}{l}\text { Stage I, with CDH repair } \\
\text { Died during combined surgery } \\
\text { Died 12 hours postoperatively } \\
\text { (poor output) }\end{array}$ \\
5 & Turner's syndrome & $45 \mathrm{XO}$ & Stage I & $\begin{array}{l}\text { Died 2 days postoperatively } \\
\text { (blocked shunt) }\end{array}$ \\
6 & Situs inversus & $46 \mathrm{XX}$ & Stage I & Well, normal development \\
& Vertebral and anorectal anomalies, & $46 \mathrm{XY}$ & Post stage I & \\
\hline
\end{tabular}

$\mathrm{CDH}$, congenital diaphragmatic hernia. 
pregnancy and subsequent management of their child. In order to make informed decisions, parents must be provided with accurate information about the different options and their respective outcomes.

This process may be affected by the concomitant diagnosis of extracardiac anomalies and chromosomal abnormalities, which were found to occur in $5 \%$ of fetuses with HLHS in our previous series..$^{12} 13$

In this centre, parents of affected babies are offered regular, non-directive counselling throughout the pregnancy by the staff of the fetal cardiology department, which includes a full time paediatric nurse counsellor. Those who choose active management are given the opportunity to meet the surgeon who will be involved in their child's care, as well as to be shown around the unit. They are given the option of elective delivery on site, to minimise the separation of mother and baby, and to avoid the risks of ex utero transfer. ${ }^{14}$

The outcome data reported here for antenatally diagnosed HLHS represents the largest series yet published, with an overall operative survival rate of $49 \%$. Comparison with the survival rate for babies who were postnatally diagnosed $(44 \%)$ is probably not valid, because of the great discrepancy in size between the two groups. Other units, with more balanced numbers of ante- and postnatally diagnosed babies, have drawn comparisons between the two groups, but with varying conclusions. One paper reported an improved outcome for infants who were diagnosed antenatally, ${ }^{9}$ attributed to optimising care in the preoperative period; two other papers reported no significant difference in outcome between ante- and postnatally diagnosed babies. ${ }^{15}{ }^{16}$ While making such comparisons, it must be borne in mind that a proportion of babies with HLHS die before reaching a cardiac centre if prenatal diagnosis is not made, ${ }^{17}$ and that postnatally diagnosed babies are therefore a selected population who have survived a collapse and transfer prior to surgery.

Differences in patient selection must also be borne in mind when comparing overall survival figures. In this centre, not only was there was a very high proportion of antenatal diagnoses, but also no baby was refused surgery because of unfavourable cardiac anatomy, or the presence of extracardiac or karyotypic anomalies. Generally, our survival figures are comparable with those of other series which have included a significant proportion of antenatal diagnoses, ${ }^{811}$ although they are lower than those in which the cases were mainly diagnosed postnatally, and/or which were more selective in their choice of cases for the Norwood operation. ${ }^{2318}$

Although mortality following stage I surgery is high, the medium term outlook for survivors is good. In our series only three of 31 children have significant neurological impairment, which may or may not have been related to previous surgery. However, there is a need for long term neurodevelopmental follow up of these children. Other reports vary in the frequency and severity of neurodevelopmental problems. The majority of children from one large series had normal verbal and performance scores, ${ }^{2}$ whereas a smaller series found that a significant number of survivors were left with major neurodevelopmental problems. ${ }^{19}$

In the long term, there is uncertainty as to how patients with HLHS will fare with a Fontan circulation, dependent on the right ventricle as the systemic pump.

Worldwide, the oldest patients are now in their late teens, and although there have been concerns about decreased exercise tolerance, ${ }^{20}$ this does not appear to be related to ventricular morphology or surgical approach. ${ }^{21}$

In conclusion, the outlook for infants with HLHS has improved dramatically over the past 20 years, and the availability of antenatal diagnosis allows parents time to come to terms with the diagnosis, and to make informed decisions. It is hoped that continuing improvements in surgical technique may not only minimise periand postoperative mortality, but reduce the risk of complications in the medium and long term.

The authors would like to thank Dr WI Norwood for his help in initiating the programme of staged surgical reconstruction for HLHS at this institution.

1 Norwood WI, Lang P, Hansen DD. Physiologic repair of aortic atresia-hypoplastic left heart syndrome. $\mathrm{N} \mathrm{Engl} \mathrm{F} \mathrm{Med}$ 1983;308:23-6.

2 Bove EL. Surgical treatment for hypoplastic left heart syndrome. Fap ₹ Thorac Cardiovasc Surg 1999;47:47-56.

3 Hehrlein FW, Yamamoto T, Orime Y, Bauer J. Hypoplastic left heart syndrome:"Which is the best operative strategy?" Ann Thorac Cardiovasc Surg 1998;4:125-32.

Ann Thorac Cardiovasc Surg 1998;4:125-32.
4 Bando K, Turrentine MW, Sun K, et al. Surgical Bando K, Turrentine MW, Sun K, et al. Surgical
management of hypoplastic left heart syndrome. Ann Thorac Surg 1996;62:70-7.

5 Hoshino K, Ogawa K, Hishitani T, et al. Hypoplastic left heart syndrome: duration of survival without surgical intervention. Am Heart f 1999;137:535-42.

6 Allan LD, Sharland GK, Tynan M. Natural history of hypoplastic left heart syndrome. Int F Cardiol 1989;25:341-3

7 Simpson J, Sharland G, Anderson D, et al. Hypoplastic left heart syndrome: willingness to initiate treatment is crucial [letter]. BMF 1997;314:1414

8 Allan LD, Apfel HD, Printz BF. Outcome after prenatal diagnosis of the hypoplastic left heart syndrome. Heart 1998;79:371-3.

9 Tworetzky W, McElhinney DB, Reddy VM, et al. Does prenatal diagnosis of hypoplastic left heart syndrome lead to improved surgical outcome? [abstract]. F $\mathrm{Am}$ Coll Cardiol 1998;31(suppl A):71A

10 Munn MB, Brumfield CG, Lau Y, Colvin EV. Prenatally diagnosed hypoplastic left heart syndrome-outcomes after postnatal surgery. $\mathcal{F}$ Matern Fetal Med 1999;8:147-50.

11 Brackley KJ, Kilby MD, Wright JG, et al. Outcome after prenatal diagnosis of hypoplastic left-heart syndrome: a case series. Lancet 2000;356:1143-7.

12 Allan LD, Sharland GK, Milburn A, et al. Prospective diagnosis of 1006 consecutive cases of congenital heart disease in the fetus. F Am Coll Cardiol 1994;23:1452-8.

13 Raymond FL, Simpson JM, Sharland GK, Mackie Ogilvie CM. Fetal echocardiography as a predictor of chromosomal abnormality [letter]. Lancet 1997;350:930.

14 Chang AC, Huhta JC, Yoon GY, et al. Diagnosis, transport, and outcome in fetuses with left ventricular outflow tract
obstruction. F Thorac Cardiovasc Surg 1991;102:841-8.

15 Kumar RK, Newburger JW, Gauvreau K, et al. Comparison of outcome when hypoplastic left heart syndrome and transposition of the great arteries are diagnosed prenatally versus when diagnosis of these two conditions is made only versus when diagnosis of these two condition
postnatally. Am 7 Cardiol 1999;83:1649-53.

16 Kern JH, Hayes CJ, Michler RE, et al. Survival and risk facKern JH, Hayes CJ, Michler RE, et al. Survival and risk fac-
tor analysis for the Norwood procedure for hypoplastic left heart syndrome. Am f Cardiol 1997;80:170-4.

17 Abu-Harb M, Hey E, Wren C. Death in infancy from unrecognised congenital heart disease. Arch Dis Child 1994;71:3-7.

18 Ishino K, Stumper O, De Giovanni JV, et al. The modified Norwood procedure for hypoplastic left heart syndrome: early to intermediate results of 120 patients with particular reference to aortic arch repair. 7 Thorac Cardiovasc Surg 1999;117:920-30.

19 Rogers BT, Msall ME, Buck GM, et al. Neurodevelopmental outcome of infants with hypoplastic left heart syndrome. 7 Pediatr 1995;126:496-8.

20 Fontan F, Kirklin JW, Fernandez G, et al. Outcome after a "perfect" Fontan operation. Circulation 1990;81:1520-36.

21 Joshi VM, Carey A, Simpson P, Paridon SM. Exercise performance following repair of hypoplastic left heart syndrome: a comparison with other types of Fontan patients. Pediatr Cardiol 1997;18:357-60. 